tine, das stumpfsinnige Schema, die Domäne der niederen Verrichtungen (wie z. B. Dokumentation), genannt Entscheidungsvorbereitung oder Entscheidungshilfe, und es gibr den unergründlichen, vom schöpferischen Menschen aus der Tiefe seines Daseins geschöpften Akt, genannt Entscheidung. Für den ersten Bereich, der noch - bei den "Trivialentscheidungen « - ein Stüdk den zweiten überdeckr, ist die Datenverarbeitung denkbar gut geeignet und sollte möglichst schnell eingesetzt werden. Im Bereich der Entscheidung allerdings, der mit dem ersten nur wenig gemein hat, im Reich der Irrationalen, kann jeder Versuch zur Rationalisierung nur Verheerungen anrichten, dort hat die Datenverarbeitung nichts verloren, da handelt es sich um Dinge, die nur von Menschen gemacht werden können.

Ganz abgesehen davon, daß diese Kategorie Entscheidung hervorragend geeignet ist, die Angst vor einer Gefährdung des Arbeitspiatzes durch Technisierung gar nicht erst aufkommen zu lassen, ermöglidht sie die oben skizzierte Haltung: man kann, ohne in Schwierigkeiten zu geraten, sowohl die Voraussetzung der eigenen Arbeit durch Datenverarbeitung umstülpen, als auch am unveränderten, handwerklichen Kern der bisherigen Arbeitsweise festhalten wollen.

Dennoch verspricht man sich von der Datenverarbeitung Großes. Man sieht die Malaise, in der gerade der hehre Bereich der Entscheidung stedst, und erhoff $\mathrm{t}$ sich Rettung von außen - eine Rettung allerdings, die das Altbewährte stabilisiert und wieder tragfähig mach - Rettung von einer Maschine, die an der Peripherie eingesetzt wird. In unserem aufgeklärten Zeitalter pfeifen auch Juristen auf den deus ex machina und richten ihre Heilserwartungen auf einen Apparat, der machina und deus in einem ist.

Dieter Rave

\title{
Empfiehlt es sich, den Deutschen Juristentag abzuschaffen?
}

"Empfiehlt es sich «, so lautet bei den Abteilungen des Juristentags die traditionelle Einleitungsformel für die Themen, die sich mit Reformvorschlägen befassen. Empfiehlt es sich also, das deutsche Rechiswesen durch die Abschaffung des Juristentags zu reformieren?

Derart ketzerische Töne zum 48. Deutschen Juristentag im September 1970 in Mainz waren nicht nur von links, sondern auch von rechts zu hören, so selbst in der FAZ, von Kühnert und Fromme, allerdings von verschiedenen Intentionen aus (25. und 26.9. 1970). Und Ingo von Münch ließ sich die Gelegenheit zu einer Glosse in der Juristenzeitung (1970, 587) nicht entgehen, die er mit Alle ( $z$ wei) Jahre wieder: der Deursche Juristentag \& überschrieb, wobei er den Leerlauf der Perfektion geißelte und sich über das $*$ Ritual von gestern $*$ lustig machte. Wenn er aber meinte, dieses Ritual würde abschrecken, so irrte er.

Die Mitgliederzahlen des Vereins und die Teilnelmerzahlen an den Juristentagen steigen rapide: 1400 in Essen (1966), 1750 in Nürnberg (1968) und jetzt iiber 3000 in Mainz. Werden die Juristen also reformfreudiger? Ist der Juristen- 
tag deshalb für die Jugend, die Referendare, deren Zahl um das Mehrfache gestiegen war, attraktiv geworden? Als aber der die Abteilung über Juristenausbildungsreform zumeist unglücklich präsidierende BGH-Senatspräsident Sarstedt den Referendaren, die den Juristentag wegen der konservativen Gutachten zur Ausbildungsreform angriffen, vorhielt: "Sie sind doch alle der Juristentag", erscholl ihm ein nahezu einstimmiges "Nein« entgegen.

Sie hatten rechr. Der Juristentag sind die anderen, die gerade das von v. Münch beklagte Ritual anzieht. Hier ist die Welt noch heil. Hier ist der unheilvolle Demokratismus noch nicht eingedrungen. Der Verein wird geleitet von einer Ständigen Deputation, die allein über Themen, Gutachter und Referenten befindet, ein Gremium, von dem nur ein Drittel alle 2 Jahre gewählt wird, gewählt nicht von den Mitgliedern, sondern von $*$ Vertrauensleuten «, die zu wählen allerdings die Mirglieder für reif erachtet werden. Hier kann sich ein untergehendes Bürgertum noch selbst feiern und bestätigen, durch die Opernfestaufführung «Die Krönung der Poppäa« wie, bei gleichsam beruhigender Langeweile, durch die Diskussion diffiziler juristischer Fragen, in dem angenehmen Gefühl, zugleich noch etwas für den Fortschritt zu tun.

Minoritäten, über die die Zeit hinweggeht, sammeln sich gern zu Heerschauen, um stark zu erscheinen, konservative Katholiken wie Vertriebene. So wird das Treffen der Juristen immer mehr zu einem Katholikentag für Juristen. Wie ein feierliches Pontifikalamt wird die "festliche Eröffnungssitzung « zelebriert, durch die traditionelle Anwesenheit des »Herrn Bundespräsidenten«, die Mitwirkung des jeweiligen Philharmonischen Orchesters, die programmatische Ansprache des Bundesministers der Justiz und durch Reden ... Reden ...

Gewiß gibr es auch progressive Mitglieder bei der Ständigen Deputation. Es ist auch zu loben, daß der bisherige Präsident Rechtsanwalt Redeker den Zusammenhang von Recht und Politik betonte, zu loben die Veranschaulichung der elektronischen Datenverarbeirung bei der Lösung von Rechrsfällen, die Vergabe des Schlußvortrags an den Chefredakreur des »Spiegel «, Günther Gaus. Und sind nicht auch fortschrittliche Beschlüsse zur Juristenausbildung, zur Ehescheidung gefaßt worden?

Aber analysieren wir diese Beschlüsse einmal. Gewiß sind in der Ausbildungsabteilung - die ganz und gar mit der Tradition langweiliger und langatmiger Diskussionsbeiträge brach und von den Referendaren nahezu in einen politischen Kongreß umfunktioniert wurde - die superkonservativen Gutachren und Referate der Professoren Oehler und Mühl sowie des Oberlandesgerichtspräsidenten Richter (fortschrittlich, aber zu abstrakt Rinken, wissenschaftlicher Assistent) vom Tisch gefegt und ist das Hamburger Modell, ein gemäßigtes Loccumer Modell, angenommen worden. Schon längst ist aber dieses Modell einer Einphasenausbildung mit Integration von Theorie und Praxis Gegenstand einer Experimentierklausel in Gesetzesentwürfen des Bundesjustizministeriums und der CDU/CSU-Bundestagsfraktion.

Uber die Vorstellungen des Bundesjustizministeriums zur Ehescheidungsreform ist die zivilrechtliche Abteilung teilweise hinausgegangen. Mir allerdings knappen Mehrheiten wurde eine Härteklausel abgelehnt, eine Trennungsfrist von nur 2 Jahren bei Widerspruch eines Ehegatten als ausreichendes Anzeichen für die Zerrüttung angesehen. Aber es handelt sich nur um eine Scheinfortschritrlichkeit, die Probleme wurden nur auf eine andere Ebene verlagert. Den zaghaften Versuchen des Bundesjustizministeriums, durch eine zeitliche Begrenzung des Unterhaltsanspruchs der geschiedenen Ehefrau ihre Eigenverantwortlichkeit bewußt zu machen, von konservativen Frauenverbänden mit Vehemenz be- 
kämpt - die Gegner der Emanzipation der Frau sind ja zumeist die Frauen selbst - wurde eine klare Absage erreilt. Die geschiedene Frau, ganz gleich, wie lange die Ehe gedauert hat und ob sie von ihr selbst »zerstört" worden ist, soll sich, wenn sie ohne Einkommen ist, immer an den Mann wenden können. Diegehässigen Auseinandersezzungen $z$ wischen den Eheleuten werden von den Scheidungs- auf die Unterhaltsprozesse verlagert, wobei gerade die geforderte weitgehende Festlegung der Scheidungsfolgen bei der Ehescheidung im Hinblick auf die nicht absehbare Zukunft der Geschiedenen Prozesse eher begünstigen dürften. Weiter zeigt auch der Ruf nach einem unbedingten Anwaltszwang, nach richterlicher Mitwirkung auch bei einverständlicher Scheidung den für Juristen typischen Zug zum Dirigismus und Perfektionismus, ist also Ausdrudk eines konservativen Juristenbildes.

Was endlich die Beschlüsse der dritten Hauptabteilung, der öffentlich-rechtlichen, zum Beamtenrecht angeht, so wäre es auch dem besten PR-Mann nicht möglich gewesen, hier etwas Fortschrittliches herauszulesen. I9I Teilnehmer sprachen sich für ein Festhalten am Berufsbeamtentum aus, bei nur 28 Gegenstimmen und I Enthaltungen; I 99 sprachen sich gegen ein Streikrecht aus, nur 45 waren dafür (I 4 Enthaltungen). Hiernach muß das Vorbereitungsgutachren des gemäßigt konservativen Professors Thieme - er wandte sich noch kürzlich gegen das Politische Mandat für Studentenschaften (JZ 1970,578) - als revolutionär linker Entwurf erscheinen.

Bedenkt man weiter den konservariven Grundzug bei der Wahl der Verrrauensleute - konservativen Professoren wurde vor progressiven, hohen Richtern vor niedrigen und weiter den Juristen des Arbeitgeberverbandes und des Industrieund Handelstages, aber nicht der Gewerkschaften das *Vertrauen " ausgesprochen -, so dürfte die im Tirel angeschnittene Frage dahin zu beantworten sein, daß die Abschaffung des Juristentags, als ein Treffen überwiegend konservativer Juristen, dem Rechtswesen dienlich sein könnte.

Gleichwohl zögern wir. Unter der Decke dieser antiquierten Honoratiorengesellschaft hat sich inzwischen so etwas wie eine Subkultur gebilder, begünstigr durch den Umstand, daß der Juristentag in dem hierarchisch strukturierten und nach der Fachomnipotenz, dem Fachidiotentum, strebenden Juristenstand nahezu der einzige Ort ist, bei dem auch zu Worr kommen kann,.wer nicht zu den Honoratioren und zu den Fachleuten gehört. Der Juristentag ist tatsächlich die Hyde Park Corner der Juristen. Natürlich stedst auch darin ein Alibi, der Ausdruck repressiver Toleranz. Aber für unser, gesellschaftlich gesehen, intolerantes Rechtswesen bedeuter schon dies einen Fortschritt. Zudem spricht der Diskussionsredner bei den großen Abteilungen in der Offentlichkeit, vor ein paar hundert Menschen, seine Worte werden protokollarisch festgehalten, die beim Juristentag stets zahlreich vertretene Presse nimmt von ihnen Kenntnis. Die Beiträge an sich liebenswürdiger Sonderlinge, die früheren Juristentagsdiskussionen einen Zug Skurrilität einfügten, werden, nicht nur in der Ausbildungsabteilung, von rechtspolitisch engagierten verdrängt. Durchweg ergab sich bei der Diskussion, auch bei der Beamtenrechtsabteilung, ein progressiveres Bild als beim Abstimmungsergebnis. Die konservative Mehrheit schwieg weitgehend und hob nur die Stimmkarte. ${ }^{1}$

1 Vgl. auch Kühnert, FAZ 25. 9. 1970 . Wenn dagegen sein Kollege Fromme - FAZ 26. 9. $1970-$ von der Linken im Wege der Abstimmung ein Umfunktionieren des Juristentags befürchter, so spiegelt dies die von Konservativen so gern zur Hilfe geholte Verschwörercheorie wieder Tatsächlich zerfallen die im Aussehen und Auftrcten sich zwar gleichenden Referendare, wie es bei der Linken üblich ist, in mehrere Richtungen, während die nichtreflektierende Rechre entschieden stärker auf Gefolgschaftstreue rechnen kann. 
Man sollte daher nur das Ergebnis der Diskussion in einem Bericht zusammenfassen, der von dem Vorsitzenden der Abteilung und zwei von der Versammlung gewählter Mitglieder erstellt wird. Dabei sollte der Juristentag sich nicht damit überfordern, zu den jeweils anstehenden Gesetzesvorhaben Stellung zu nehmen, sondern sich stärker mit Strukturproblemen des Rechtswesens befassen, z. B. Theorie und Praxis richterlichen Handelns, wie ja die Ausbildungsabteilung beschlossen hatte. Ein anderes Problemgebiet wären die Allgemeinen $\mathrm{Ge}-$ schäftsbedingungen in einer kapitalistischen Wirtschaft. Alsdann läge es auf der Hand, Gutachten an Arbeitsgruppen zu vergeben, wie ebenfalls die Ausbildungsabteilung beschlossen hatte, wobei Gesellschaftswissenschaftler hinzuzuziehen wären.

Hierdurch würde der Juristentag mehr den Charakter einer Arbeitstagung bekommen und alsdann, bei weitgehendem Abbau des Rahmenprogramms $\rightarrow$ für die Damen«, auch in der Zahl der Teilnehmer wieder übersehbar werden. Natürlich müßte sich auch die Ständige Deputation jedem Juristentag ohne $Z_{\text {wi- }}$ schenschaltung von Vertrauensleuten zur Wahl stellen. Allerdings wird dabei die Deputation bei den derzeitigen Mehrheitsverhältnissen - zumal die Zahl der Referendare erheblich absinken mag, wenn kein Ausbildungsthema angeboten wird - noch konservativer werden. Das kann indes in Kauf genommen werden, sofern institutionell abgesichert ist, da $B$ in den Abreilungen das Arbeits- und nicht das Stimmenergebnis zählt.

Sollte aber eine konservativere Ständige Deputation erst recht in der Tradition verharren, um Juristentage zu Bekenntnistagen für das abgestandene Ordnungsbild des bürgerlichen Juristen, für den Primat eines antiquitierten juristischen Dogmatismus hochzustilisieren, so dürfte auch das den Klärungsprozeß in der Gesellschaft erfreulich beschleunigen. Es zeigte sich nämlich dann, daß der Jurist in einer fortschrittlichen, der Offenheit zugehenden Gesellschaft keinen Platz mehr besitzt.

Theo Rasehorn

\section{Juristenphilosophie}

Es tagte die bundesdeutsche Sektion der Internationalen Vereinigung für Rechtsund Sozialphilosophie Anfang Oktober in Freiburg i. Br. Zum Thema $>$ Wozu Rechtsphilosophie heute؛ wollten Juristen sich äußern. Sie produzierten Juristenphilosophie. ${ }^{1}$

Unter Werner Maihofers gemäßigt zentralistischer Ägide wurde Philosophieren zum Ereignis - und was sich da ereignete: in einem Referat wurde zugleich die

\footnotetext{
' Das eher gedankenios als pluralistisch zusammengestellice Programm umfaßre die Themen: Wozu Rechtsphilosophie heute? (Arthur Kaufmann); Die Funktion der Rechtsphilosophie in der Theorie des Rechts (Marcic, Ryffel, Zippelius); Rechtsphilosophie als Kritik der Rechtswissenschaft (Ebbinghaus, Viehweg); Materialistische Philosophie und Sozizlistische Theorie des Rechts (W. R. Beyer); Rechrsphilosophie in Forschung und Lehre (Denninger, Naudse, Pawlowski); Die Funkrion der Rechtsphilosophie in der Praxis des Rechts (Roelledke, Dreicr, Häberle, E. ๆ. Savigny).
} 